\title{
Radiological chest manifestations in diffuse infiltrative lymphocytosis syndrome (DILS)
}

\section{F Ismail, FCRad(D) \\ Irma van de Werke, FRCR \\ Nausheen Khan, FCRad (D)}

Department of Radiology, Kalafong Hospital, Pretoria

P F Levay, MSC, MB ChB, MMed (Int)

Department of Internal Medicine, Kalafong Hospital

\section{G H Davel, MMed (Anat Path)}

Department of Anatomical Pathology, Kalafong Hospital

Corresponding author: F Ismail (drfismail@yahoo.com)
Lymphocytic interstitial pneumonitis (LIP) is part of the DILS spectrum. ${ }^{2}$ LIP is a rare disease which is more common in children and the radiological presentation of DILS in adults is similar to LIP in children.

\section{Imaging findings}

The lung is the most commonly affected extra-glandular site of DILS. The disease may mimic Pneumocystis jirovecii pneumonia with bilateral

\section{Introduction}

Diffuse infiltrative lymphocytosis syndrome (DILS) is an entity found in patients infected with the human immune deficiency virus (HIV) with a persistent CD8+ lymphocytosis and diffuse lymphocytic infiltration of various organs. ${ }^{1}$ The aetiology is unknown, but it is postulated that the possible cause is a 'hyper-immune' lung response to HIV or Epstein-Barr virus that results in infiltration of organs by the CD8+ lymphocyte. ${ }^{2}$

Typically, patients present with asymptomatic salivary gland enlargement. However, in our setting (third-world), patients may not necessarily present in this way. Other features include sicca or Sjogrenslike syndrome and associated cervical and generalised lymphadenopathy. ${ }^{1}$ Unilateral parotid gland enlargement in an HIV-positive patient should alert the clinician to suspected DILS. ${ }^{3}$ Radiological manifestations of this disease should be first sought in the lungs, which is the most common extra-glandular site of infection. Other manifestations of this entity include a severe form of peripheral neuropathy, hepatitis, myositis and nephritis. ${ }^{3}$

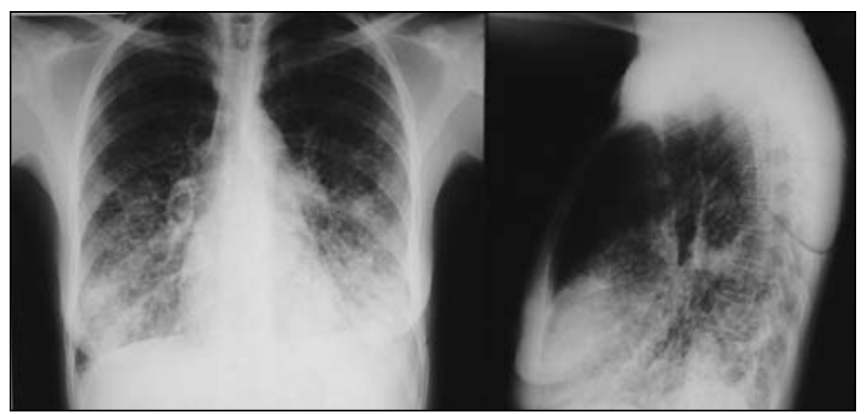

Fig. 1. Frontal and lateral chest radiographs in an adult female patient demonstrate bilateral basal reticulo-nodular infiltrate with areas of coalescence in both lower lobes, and lingula resulting in areas of subsegmental consolidation. Hilar adenopathy is present.

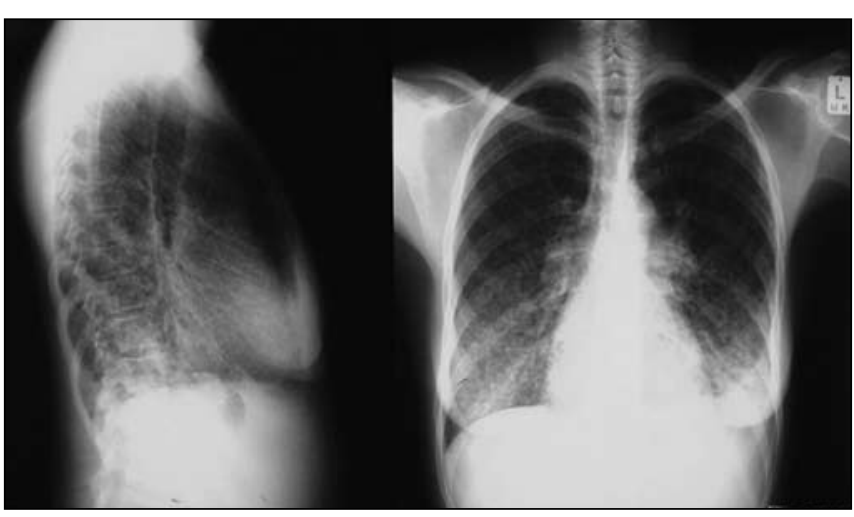

Fig. 2. Frontal and lateral radiographs of an adult female patient demonstrate predominantly nodular infiltrate distributed in the basal lung zones with hilar adenopathy and left pleural effusion.

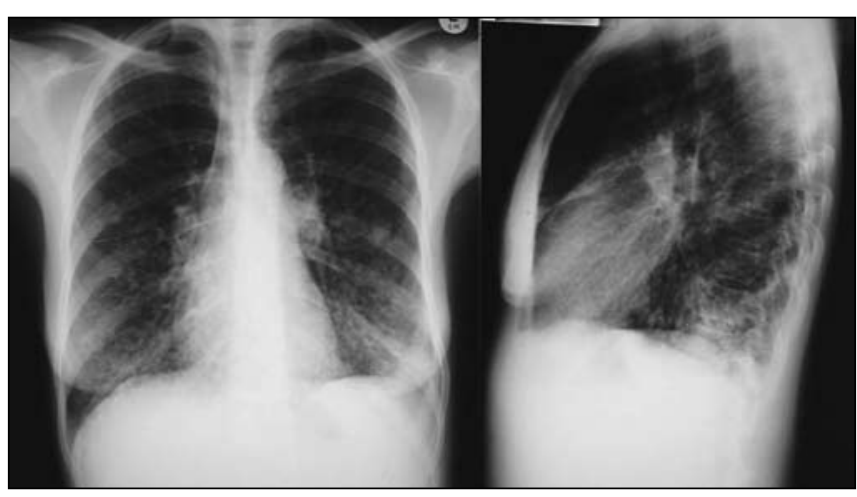

Fig. 3. Frontal and lateral chest radiographs demonstrate coarse reticular and nodular infiltrate in the mid and lower lung zones, with associated hilar adenopathy and left basal consolidation. 


\section{CASE REPORT}
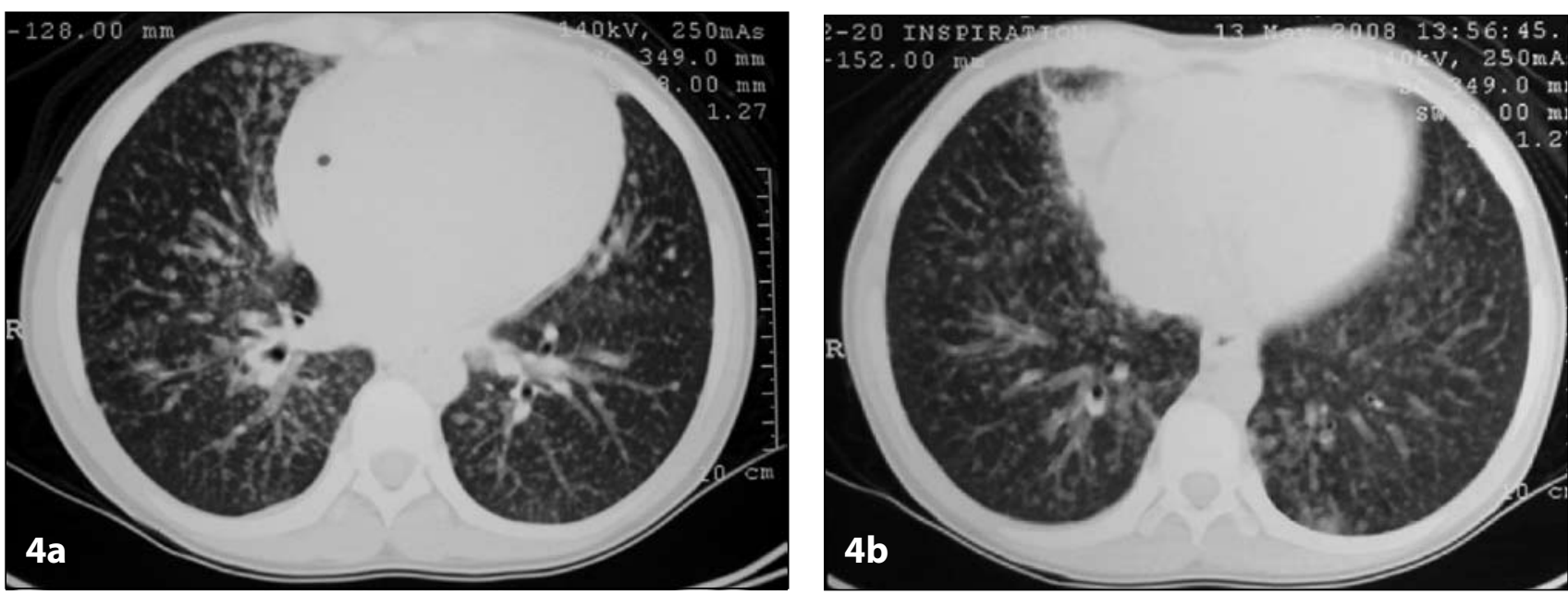

Figs $4 a$ and b. Axial high resolution computed tomography (HRCT) of the chest demonstrates lymphatic distribution of a nodular infiltrate with interlobular septal thickening.
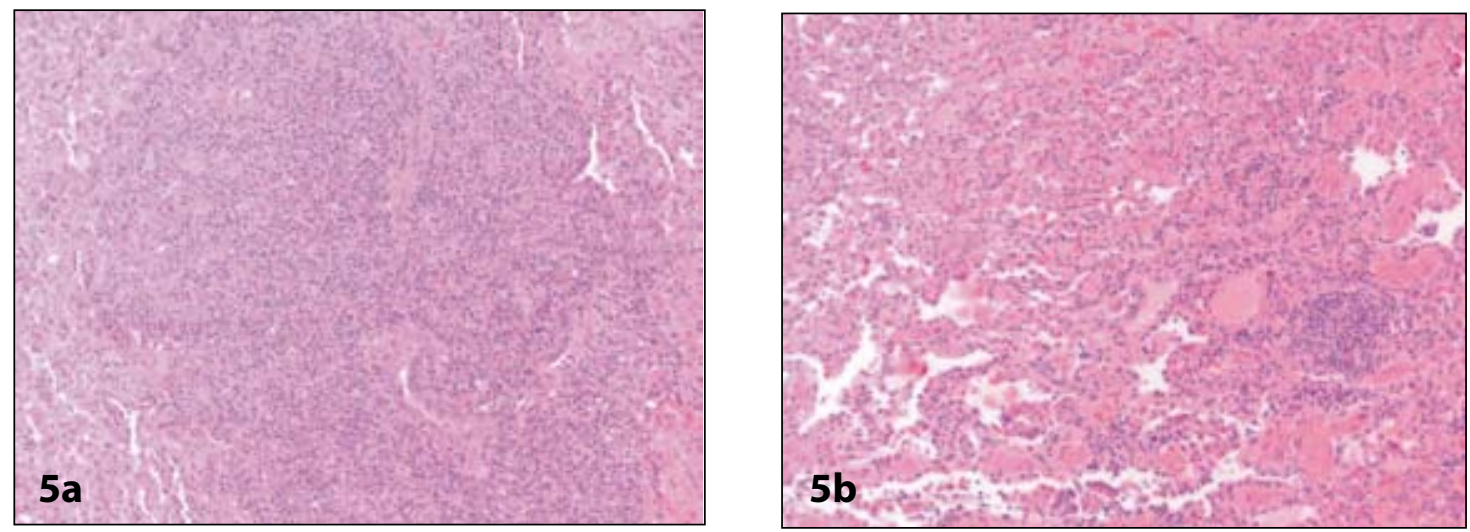

Figs $5 a$ and $b$. Histological stains of lung biopsy demonstrate thickening of the interalveolar septae with focal peribronchiolar collections of lymphocyte infiltration in keeping with interstitial lung disease.

ground-glass opacification and reticulonodular infiltrate. It may also be confused with miliary TB as demonstrated on chest radiograph (Figs 1 - 3) and high-resolution computed tomography images (Fig. 4).

As bilateral hilar adenopathy and basal Kerley B lines are also a feature, lymphangitis carcinomatosis and lymphoma of the lung are also possible differential diagnoses to consider. The diagnosis is confirmed histologically (Fig. 5) by demonstrating diffuse lymphocytic infiltration of the lung. Treatment involves the use of anti-retrovirals and steroids.

\section{Recommendation}

Radiologists should consider DILS as part of the differential diagnosis in HIV-positive patients who present with enlarged parotid glands and a basal miliary infiltrate with hilar adenopathy, and should timeously alert the clinician of this entity.

1. Levay PF, Botes ME. Diffuse infiltrative lymphocytosis syndrome. SA Fam Pract 2008;50(2):23-27.

2. Jeanes AC, Owens DM. Imaging of HIV disease in children. Imaging 2002;14:8-23.

3. Franco-Paredes C, Robolledo P, Folch E, Hernandez I, del Rio C. Diagnosis of diffuse CD8+ lymphocytosis syndrome in HIV infected patients. AIDS Reader 2002;12(9):410-411. 